\title{
On the complexity of single machine scheduling problems under scenario-based uncertainty ${ }^{1}$
}

\author{
Mohamed Ali Aloulou*, Federico Della Croce ${ }^{\dagger}$
}

\begin{abstract}
Résumé
Nous considérons des problèmes d'ordonnancement où certains paramètres des tâches sont incertains. Cette incertitude est modélisée au travers d'un ensemble fini de scénarios bien définis. Nous cherchons une solution qui soit acceptable pour l'ensemble des scénarios considérés. Plusieurs critères ont été utilisés dans la littérature pour sélectionner la "meilleure" solution. Nous utilisons ici le critère appelé robustesse absolue. Nous présentons des résultats algorithmiques et de complexité pour quelques problèmes classiques d'ordonnancement sur une machine.
\end{abstract}

Mots-clefs : Ordonnancement ; Données incertaines ; Scénarios ; Robustesse absolue

\begin{abstract}
We consider scheduling environments where some job characteristics are uncertain. This uncertainty is modeled through a finite set of well-defined scenarios. In such a context, we search for a solution that is acceptable for any considered scenario. For this purpose, several criteria can be applied to select among solutions. We use here the so-called absolute robustness criterion. We present algorithmic and computational complexity results for several single machine scheduling problems.
\end{abstract}

Key words : Scheduling; Scenario-based uncertainty; Absolute robustness.

\footnotetext{
${ }^{1}$ Research performed while the second author was visiting LAMSADE on a research position funded by the CNRS

†LAMSADE, Université Paris-Dauphine, 75775 Paris cedex 16, France. al ou lou@ lamsade. dauphine.fr

${ }^{\ddagger}$ D.A.I., Politecnico di Torino, Italy. federico.dellacroce@polito.it
} 


\section{Introduction}

This paper deals with single machine scheduling problems where some job characteristics are uncertain. This uncertainty is described through a finite set $S$ of well-defined scenarios. We denote by $p_{j}^{s}, d_{j}^{s}$ and $w_{j}^{s}$, respectively, the processing time, the release date, the due date and the weight of job $j$ under scenario $s \in S$.

Consider a scheduling problem, denoted by $\alpha|\beta| \gamma$ according to Graham et al. notation [5]. Let $\Pi$ be the set of feasible schedules with respect to the problem constraints. For each scenario $s \in S$, we denote by $\operatorname{OPT}(\alpha|\beta| \gamma, s)$ the problem of finding an optimal schedule $\pi_{s}^{*}$ satisfying

$$
F\left(\pi_{s}^{*}, s\right)=\min _{\pi \in \Pi} F(\pi, s)
$$

When problem parameters are uncertain, it is appropriate to search for a solution that is acceptable for any considered scenario. For this purpose, several criteria can be applied to select among solutions. In [8], Kouvelis and Yu proposed three different robustness criteria: the absolute robustness or maximal cost, the maximal regret or robust deviation and the relative robustness. In this paper, we focus on the absolute robustness criterion.

To the best of our knowledge, the absolute robustness in single machine scheduling problems has only been considered in [3] and [12] where, for the 1\| $\sum C_{j}$ problem with uncertain processing times, two distinct proofs of the $N P$-hardness even for $|S|=2$ were provided (notice that the corresponding deterministic version is well known to be polynomially solvable [11]). The maximal regret criterion was instead much more studied (see for instance, again [12] but also [1, 6, 7]).

The absolute robustness of schedule $\pi$ over all scenarios $s \in S$ is denoted by $\bar{F}(\pi)$. We have

$$
\bar{F}(\pi)=\max _{s \in S} F(\pi, s) .
$$

We denote by $\operatorname{Min} \operatorname{Max}(\alpha|\beta| \gamma, \theta)$ the problem of finding a schedule $\pi^{A}$ minimizing the absolute robustness $\bar{F}(\pi)$ among all schedules $\pi \in \Pi$. Field $\theta$ indicates the set of uncertain problem parameters. For the problems considered here, $\theta \subseteq\left\{p_{j}, d_{j}, w_{j}\right\}$. Sequence $\pi^{A}$ is called in [8] absolute robust sequence. Its $\operatorname{cost} \bar{F}\left(\pi^{A}\right)$ satisfies

$$
\bar{F}\left(\pi^{A}\right)=\min _{\pi \in \Pi} \bar{F}(\pi)=\min _{\pi \in \Pi} \max _{s \in S} F(\pi, s) .
$$

Notice that if problem $\alpha|\beta| \gamma$ is NP-hard, then, in the presence of uncertainty, the corresponding problems $\operatorname{Min} \operatorname{Max}(\alpha|\beta| \gamma, \theta)$ are also NP-hard. However, if problem $\alpha|\beta| \gamma$ is polynomially solvable, then, the corresponding problems $\operatorname{Min} \operatorname{Max}(\alpha|\beta| \gamma, \theta)$ are not necessarily polynomially solvable. In this work we establish the complexity status for 
the absolute robustness versions of the most well known non-preemptive polynomialtime single machine scheduling problems, namely problems $1 \mid$ prec $\mid f_{\max }$ (with $f_{\max } \in$ $\left.\left\{C_{\max }, L_{\max }, T_{\max }\right\}\right), 1 \| \sum w_{j} C_{j}$ and $1 \| \sum U_{j}$. Notice that all these problems present regular cost functions non-decreasing in the job completion times. In this context any schedule $\pi \in \Pi$ is completely characterized by the corresponding job sequence. Given a schedule $\pi \in \Pi$, the completion time of job $j$ under scenario $s$, denoted by $C_{j}(\pi, s)$, $j=1, \ldots, n, s \in S$, can easily be determined and the quality of the schedule $\pi \in \Pi$ under scenario $s$ is then evaluated using the regular cost function $F(\pi, s)$. We consider the following cost functions :

- the general maximum cost function $f_{\max }=\max _{j}\left\{f_{j}\left(C_{j}\right)\right\}$ with $f_{\max } \in\left\{C_{\max }\right.$, $\left.L_{\max }, T_{\max }\right\}$ : hence we deal with the maximum completion time (makespan) $C_{\max }=$ $\max _{j}\left\{C_{j}\right\}$, the maximum lateness $L_{\max }=\max _{j}\left\{L_{j}\right\}$ with $L_{j}=C_{j}-d_{j}$ and the maximum tardiness $T_{\max }=\max _{j}\left\{T_{j}\right\}$ with $T_{j}=\max \left\{0, L_{j}\right\}$;

- the total weighted completion time $\sum_{j} w_{j} C_{j}$;

- the number of late jobs $\sum_{j} U_{j}$ with $U_{j}=0$, if job $j$ is on-time $\left(C_{j} \leq d_{j}\right)$ and $U_{j}=1$ if $j$ is late $\left(C_{j}>d_{j}\right)$.

Using the set $S$ of scenarios, we construct a scenario $s^{w}$ in which parameters $k_{j}$ take their worst case value, denoted by $k_{j}^{w}$. In our case, we have $p_{j}^{w}=\max _{s \in S} p_{j}^{s}$, $d_{j}^{w}=$ $\min _{s \in S} d_{j}^{s}$ and $w_{j}^{w}=\max _{s \in S} w_{j}^{s}$. Notice that in the context of a discrete set of scenarios, the constructed scenario is not necessarily feasible, i.e. we can have $s^{w} \notin S: s^{w}$ is called worst-case artificial scenario.

Remark 1. When parameters are interval-uncertain, $s^{w}$ is a feasible scenario. In this case, an absolute robust solution $\pi^{A}$ of problem $\operatorname{Min} \operatorname{Max}(1|\beta| \gamma, \theta)$ is such that

$$
\begin{aligned}
\bar{F}\left(\pi^{A}\right)=\min _{\pi \in \Pi} \bar{F}(\pi) & =\min _{\pi \in \Pi} \max _{s \in S} F(\pi, s) \\
& =\min _{\pi \in \Pi} F\left(\pi, s^{w}\right) .
\end{aligned}
$$

Hence $\pi^{A}$ is also optimal for problem $O P T\left(1|\beta| \gamma, s^{w}\right)$. This means that the problem of finding an absolute robust sequence can be solved straigtforwardly by the algorithm solving the problem without uncertainty applied to the worst-case artificial scenario.

When uncertainty is scenario-based, we can not apply the same reasoning because scenario $s^{w}$ is not necessarily feasible. Nevertheless, we show in this paper that problems $\operatorname{MinMax}\left(1 \mid\right.$ prec $\left.\mid L_{\max }, d_{j}\right)$ and MinMax $\left(1|| \sum U_{j}, d_{j}\right)$ can be solved by Lawler's algorithm [9] and Moore's algorithm [10] respectively applied to the worst case artificial 
scenario. We also prove that an extension of Lawler's algorithm, called here MinMaxLawler, solves problem MinMax $\left(1 \mid\right.$ prec $\left.\mid f_{\text {max }}, p_{j}, d_{j}\right)$ in polynomial time. On the other hand, problems $\operatorname{Min} \operatorname{Max}\left(1|| \sum w_{j} C_{j}, w_{j}\right)$ and $\operatorname{Min} \operatorname{Max}\left(1 \| \sum U_{j}, p_{j}\right)$ are proved to be NP-hard even when $|S|=2$.

Table 1 summarizes the above results presenting the complexity status for the absolute robustness versions of the most well known non-preemptive polynomial-time single machine scheduling problems, where an entry "-" indicates that the considered case is not applicable (for instance problem $1 \| \sum w_{j} C_{j}$ cannot have uncertainty on due dates as due dates are not present in the problem).

Table 1: Summary of the obtained results

\begin{tabular}{|c|c|c|c|}
\hline \multirow{2}{*}{$\begin{array}{c}\text { Uncertain } \\
\text { parameter }\end{array}$} & \multicolumn{3}{|c|}{ cost function } \\
\cline { 2 - 4 } & $1 \| \sum w_{j} C_{j}$ & $1 \mid$ prec $\mid f_{\max }$ & 1||$\sum U_{j}$ \\
\hline$d_{j}$ & - & $O\left(n^{2}+n|S|\right)($ Th. 1) & $O(n \log n+n|S|)($ Th. 4) \\
\hline$p_{j}$ & NP-hard [3, 12] & $O\left(n^{2}|S|\right)$ (Th. 2) & NP-hard (Th. 5) \\
\hline$w_{j}$ & NP-hard (Th. 3) & - & - \\
\hline$p_{j}$ and $d_{j}$ & - & $O\left(n^{2}|S|\right)$ (Coro. 2) & NP-hard (Coro. 3) \\
\hline$p_{j}$ and $w_{j}$ & NP-hard [3, 12] & - & - \\
\hline
\end{tabular}

\section{Problem $\operatorname{MinMax}\left(1 \mid\right.$ prec $\left.\mid f_{\max }, \theta\right)$}

\subsection{Uncertainty on due dates}

We consider problem MinMax $\left(1 \mid\right.$ prec $\left.\mid f_{\max }, d_{j}\right)$ where processing times are deterministic and due dates are uncertain (here $f_{\max } \in\left\{L_{\max }, T_{\max }\right\}$ as for $C_{\max }$ no uncertainty holds). In this case the worst-case artificial scenario $s^{w}$ is such that, for all $j \in N$, $d_{j}^{w}=\min _{s \in S} d_{j}^{s}$.

We recall that problem $1 \mid$ prec $\mid f_{\max }$ can be solved in $O\left(n^{2}\right)$ time by Lawler's algorithm [9]. This algorithm constructs an optimal schedule backwards. At the points in time where the unscheduled jobs should complete, starting with point $t=P=\sum_{j \in N} p_{j}$, Lawler's algorithm chooses among the unscheduled jobs having no successors a job with minimum cost to be completed at $t$. Notice that as processing times are deterministic, we have

$$
\forall \pi \in \Pi, \forall s \in S, \forall j \in N, C_{j}(\pi, s)=C_{j}(\pi) .
$$

The following theorem holds.

Theorem 1. Problem MinMax $\left(1 \mid\right.$ prec $\left.\mid f_{\max }, d_{j}\right)$ can be optimally solved in $O\left(n^{2}+n|S|\right)$ time by means of Lawler's algorithm applied to the worst-case artificial scenario $s^{w}$. 
Proof. In the following, for the sake of clarity we consider that $f_{\max }=L_{\max }$ but the same analysis holds for $f_{\max }=T_{\max }$. An absolute robust solution $\pi^{A}$ of problem $\operatorname{MinMax}\left(1 \mid\right.$ prec $\left.\mid L_{\max }, d_{j}\right)$ is such that

$$
\begin{aligned}
\bar{L}\left(\pi^{A}\right) & =\min _{\pi \in \Pi} \max _{s \in S} L_{\max }(\pi, s) \\
& =\min _{\pi \in \Pi} \max _{s \in S} \max _{j \in N}\left(C_{j}(\pi, s)-d_{j}^{s}\right) \\
& =\min _{\pi \in \Pi} \max _{s \in S} \max _{j \in N}\left(C_{j}(\pi)-d_{j}^{s}\right) \\
& =\min _{\pi \in \Pi} \max _{j \in N} \max _{s \in S}\left(C_{j}(\pi)-d_{j}^{s}\right) \\
& =\min _{\pi \in \Pi} \max _{j \in N}\left(C_{j}(\pi)-d_{j}^{w}\right) \\
\bar{L}\left(\pi^{A}\right) & =\min _{\pi \in \Pi} L_{\max }\left(\pi, s^{w}\right)
\end{aligned}
$$

Hence, $\pi^{A}$ is also an optimal solution for problem OPT $\left(1 \mid\right.$ prec $\left.\mid L_{\max }, s^{w}\right)$. For the complexity, the construction of the worst-case scenario requires $O(n|S|)$ time and the application of Lawler's algorithm requires $O\left(n^{2}\right)$ time, hence the overall complexity is $O\left(n^{2}+n|S|\right)$.

We observe that the proof of Theorem 1 can be applied, as it is, to any scheduling problem $\alpha|\beta| f_{\max }$. Hence, we have the following result.

Corollary 1 Any algorithm optimally solving problem $\alpha|\beta| f_{\max }$ provides an absolute robust solution for problem MinMax $\left(\alpha|\beta| f_{\max }, d_{j}\right)$, when applied to the worst-case artificial scenario $s^{w}$.

\subsection{Uncertainty on processing times and due dates}

We consider problem $\operatorname{MinMax}\left(1 \mid\right.$ prec $\left.\mid f_{\max }, p_{j}, d_{j}\right)$ where we suppose now that both processing times and due dates are uncertain. A robust solution $\pi^{A}$ is such that

$$
\begin{aligned}
\bar{F}\left(\pi^{A}\right) & =\min _{\pi \in \Pi} \max _{s \in S} F_{\max }(\pi, s) \\
& =\min _{\pi \in \Pi} \max _{s \in S} \max _{j \in N} f_{j}\left(C_{j}(\pi, s)\right)
\end{aligned}
$$

We propose an algorithm, called MinMax-Lawler, which is an extension of Lawler's algorithm. This algorithm constructs a sequence $\pi$ in reverse order. Let $U$ be the set of unscheduled jobs. Define $p^{s}(U)=\sum_{j \in U} p_{j}^{s}$ for all $s \in S$. The rule is the following : Schedule last the job $j \in U$, which has no successor in $U$ and such that $\max _{s \in S} f_{j}^{s}\left(p^{s}(U)\right)$ is minimal. It is immediate to see that the complexity of MinMax-Lawler is $O\left(n^{2}|S|\right)$.

We have the following result. 
Theorem 2. Problem MinMax $\left(1 \mid\right.$ prec $\left.\mid f_{\max }, p_{j}, d_{j}\right)$ is optimally solved by algorithm MinMax-Lawler.

Proof. The proof is very similar to the proof of Lawler's algorithm optimality for problem $1 \mid$ prec $\mid f_{\max }$.

Enumerate the jobs in such a way that $(1,2, \ldots, n)$ is the sequence constructed by the proposed algorithm. Let $\pi^{A}$ be an absolute robust sequence for problem $\operatorname{MinMax}\left(1 \mid\right.$ prec $\left.\mid f_{\max }, p_{j}, d_{j}\right)$ with $\pi^{A}(i)=i$ for $i=n, n-1, \ldots, r$ and $\pi^{A}(r)=j<r$.

Notice that it is possible to schedule $r-1$ immediately before $r$. Hence, we can construct a sequence $\pi^{\prime}$ in which we shift to the left the block between jobs $r-1$ and $r$ and process $r-1$ immediately before $r$. Clearly,

$$
\forall i \in N-\{r-1\}, \forall s \in S, C_{i}\left(\pi^{\prime}, s\right) \leq C_{i}\left(\pi^{A}, s\right)
$$

Hence,

$$
\begin{aligned}
\bar{F}\left(\pi^{\prime}\right)=\max _{s \in S} F\left(\pi^{\prime}, s\right) & =\max \left\{\bar{F}\left(\pi^{A}\right), \max _{s \in S} f_{r-1}^{s}\left(C_{r-1}\left(\pi^{\prime}, s\right)\right)\right\} \\
& =\max \left\{\bar{F}\left(\pi^{A}\right), \max _{s \in S} f_{r-1}^{s}\left(C_{j}\left(\pi^{A}, s\right)\right)\right\} \\
& \leq \max \left\{\bar{F}\left(\pi^{A}\right), \max _{s \in S} f_{j}^{s}\left(C_{j}\left(\pi^{A}, s\right)\right)\right\} \\
& \leq \bar{F}\left(\pi^{A}\right)
\end{aligned}
$$

Consequently $\pi^{\prime}$ is also an absolute robust sequence.

We can reiterate the same reasoning and transform sequence $\pi^{A}$ into sequence $(1,2, \ldots, n)$ without increasing the objective function value.

Correspondingly. the following corollary also holds.

Corollary 2 Problem MinMax $\left(1 \mid\right.$ prec $\left.\mid f_{\max }, p_{j}\right)$ is optimally solved by algorithm MinMax-Lawler.

\section{Problem $\operatorname{MinMax}\left(1 \| \sum w_{j} C_{j}, w_{j}\right)$}

We consider problem $\operatorname{MinMax}\left(1 \| \sum w_{j} C_{j}, w_{j}\right)$ where processing times are deterministic and weights are uncertain. We prove that this problem is $N P$-hard even when $|S|=2$ and $p_{j}=1 \forall j$. To this extent we need to prove the following instrumental lemma. 
Lemma 1. The $1 \| \sum C_{j}$ problem and the $1\left|p_{j}=1\right| \sum w_{j} C_{j}$ problem are equivalent.

Proof. Given any instance of the $1 \| \sum C_{j}$ problem where each job $j$ has processing time $p_{j}^{\prime}$, generate an instance of the $1\left|p_{j}=1\right| \sum w_{j} C_{j}$ problem where each job $j$ has weight $w_{j}^{\prime \prime}=p_{n-j+1}^{\prime}$. Consider a generic sequence $(1,2, \ldots, n-1, n)$. For the $1 \| \sum C_{j}$ problem the corresponding cost function value is $Z_{1}=\sum_{j=1}^{n}(n-j+1) p_{j}^{\prime}$. For the $1 \mid p_{j}=$ $1 \mid \sum w_{j} C_{j}$ problem the corresponding cost function value is $Z_{2}=\sum_{j=1}^{n} j w_{j}^{\prime \prime}$. We show that $Z_{2}=Z_{1}$. Indeed, $Z_{2}=\sum_{j=1}^{n} j w_{j}^{\prime \prime}=\sum_{j=1}^{n} j p_{n-j+1}^{\prime}=\sum_{j=1}^{n}(n-j+1) p_{j}^{\prime}=Z_{1}$.

Theorem 3. Problem MinMax $\left(1|| \sum w_{j} C_{j}, w_{j}\right)$ is NP-hard even when $|S|=2 p_{j}=$ $1 \forall j$.

Proof. Due to Lemma 1 and the $N P$-hardness of problem $\operatorname{MinMax}\left(1 \| \sum C_{j}, p_{j}\right)$ from $[3,12]$, the proof immediately holds.

\section{Problem MinMax $\left(1 \| \sum U_{j}, \theta\right)$}

We consider problem $\operatorname{Min} \operatorname{Max}\left(1 \| \sum U_{j}, \theta\right)$ with $\theta \subseteq\left\{p_{j}, d_{j}\right\}$. We show that problem $\operatorname{MinMax}\left(1 \| \sum U_{j}, d_{j}\right)$ can be solved by Moore's algorithm [10] applied to the worstcase artificial scenario, whereas problem $\operatorname{Min} \operatorname{Max}\left(1 \| \sum U_{j}, p_{j}\right)$ is NP-hard.

\subsection{Uncertainty on due dates}

Lemma 2. There exist an optimal solution of problem MinMax $\left(1 \| \sum U_{j}, d_{j}\right)$ in which on-time jobs are scheduled in a non-decreasing order of their worst-case artificial scenario due dates $d_{j}^{w}$.

Proof. Let $\pi^{A}$ be an optimal solution for problem $\operatorname{MinMax}\left(1 \| \sum U_{j}, d_{j}\right)$. Suppose that in this schedule there exist two consecutive on-time jobs $i$ and $j$ such that $i \prec j$ and $d_{i}^{w} \geq d_{j}^{w}$. We have

$$
\forall s \in S, C_{i}\left(\pi^{A}, s\right)=C_{i}\left(\pi^{A}\right) \leq d_{i}^{s}
$$

and

$$
\forall s \in S, C_{j}\left(\pi^{A}, s\right)=C_{j}\left(\pi^{A}\right) \leq d_{j}^{s}
$$

By interchanging jobs $i$ and $j$, we obtain a new feasible schedule denoted by $\pi$. In this schedule, only completion time of job $i$ increases and we have

$$
C_{i}(\pi)=C_{j}\left(\pi^{A}\right)
$$


According to equation (21), we obtain

$$
C_{i}(\pi) \leq \max _{s \in S} d_{j}^{s} \leq d_{j}^{w} \leq d_{i}^{w} \leq d_{i}^{s}, \forall s \in S
$$

This means that job $i$ is on-time in schedule $\pi$ and consequently $\pi$ is also optimal for problem MinMax $\left(1 \| \sum U_{j}, d_{j}\right)$.

We can reiterate the same reasoning and obtain finally a solution in which on-time jobs are sequenced in a non-decreasing order of their worst-case artificial scenario due dates $d_{j}^{w}$.

Theorem 4. Problem MinMax $\left(1 \| \sum U_{j}, d_{j}\right)$ can be optimally solved in $O(n \log n+$ $n|S|)$ time by means of Moore's algorithm applied to the worst-case artificial scenario $s^{w}$.

Proof. The proof is very similar to the proof of Moore's algorithm optimality for problem $1 \| \sum U_{j}$.

According to Lemma 2, there exists an optimal schedule $\pi=\left(\pi_{I}, \pi_{L}\right)$ for problem $\operatorname{MinMax}\left(1 \| \sum U_{j}, d_{j}\right)$ in which

- on-time jobs are scheduled before late jobs, and,

- on-time jobs are scheduled according to the non-decreasing order of their worstcase artificial scenario due dates $d_{j}^{w}$.

Without loss of generality, enumerate the jobs such that $d_{1}^{w} \leq d_{2}^{w} \leq \ldots \leq d_{n}^{w}$. Let $j$ be the first job deleted, in Moore's algorithm, from the set $I$ of on-time jobs. Suppose that job $j$ is on-time in $\pi$. We transform $\pi$ in another optimal schedule in which $j$ is late. Remark that there exists at least one job $k$, with $k<j$, which is late in $\pi$. Otherwise, Moore's algorithm would not have rejected $j$. It is immediate to prove that by interchanging jobs $j$ and $k$ we get a new optimal schedule in which job $j$ is late. We can reiterate the same reasoning and transform then sequence $\pi^{A}$ into the sequence obtained by Moore's algorithm applied to the worst-case artificial scenario $s^{w}$ without increasing the number of late jobs.

For the complexity, the construction of the worst-case scenario requires $O(n|S|)$ time and Moore's algorithm requires $O(n \log n)$ time, hence the overall complexity is $O(n \log n, n|S|)$. 


\subsection{Uncertainty on processing times}

We prove that problem $\operatorname{Min} \operatorname{Max}\left(1 \| \sum U_{j}, p_{j}\right)$ is NP-hard even when $|S|=2$ by constructing a reduction from the NP-complete even-odd partition problem [4].

Even-odd partition problem: Given $2 n$ positive integers $a_{1}<a_{2}<\ldots<a_{2 n}$ where $\sum_{j=1}^{2 n} a_{j}=2 B$, is there a partition of the integers into two subsets $A_{1}$ and $A_{2}$ such that

$$
\sum_{j \in A_{1}} a_{j}=\sum_{j \in A_{2}} a_{j}=B
$$

and $A_{1}$ and $A_{2}$ each contains exactly one element of each pair $\left(a_{2 i-1}, a_{2 i}\right), i=1, \ldots, n$ ?

Given an instance of the even-odd partition problem, we construct the following instance of problem $\operatorname{MinMax}\left(1 \| \sum U_{j}, p_{j}\right)$.

Instance of problem $\operatorname{Min} \operatorname{Max}\left(1 \| \sum U_{j}, p_{j}\right)$ : We have $2 n$ jobs and two scenarios 1 and 2. Job processing times and due dates are such that

$$
\begin{gathered}
\qquad\left\{\begin{array}{l}
p_{1}^{1}=p_{2}^{2}=a_{1} \\
p_{1}^{2}=p_{2}^{1}=a_{2} \\
d_{1}=d_{2}=a_{2}
\end{array}\right. \\
\forall i=2, \ldots, n\left\{\begin{array}{l}
p_{2 i-1}^{1}=p_{2 i}^{2}=a_{2 i-1}+a_{2 i-2}+\ldots+a_{2} \\
p_{2 i-1}^{2}=p_{2 i}^{1}=a_{2 i}+a_{2 i-2}+\ldots+a_{2} \\
d_{2 i-1}=d_{2 i}=a_{2 i}+2 a_{2 i-2}+\ldots+i a_{2}, \text { for } i<n, \text { and }, \\
d_{2 n}=d_{2 n-1}=B+d_{2 n-2}
\end{array}\right.
\end{gathered}
$$

Jobs $2 i-1$ and $2 i$ are called $a_{[i]}-j o b s$. Notice that with this construction, the following lemmata hold.

Lemma 3. Any sequence in which each position $i, i=1, \ldots, n$, is occupied by an $a_{[i]}-j o b$ admits at least $n-1$ on-time jobs.

Proof. We can prove straightforwardly that in such a sequence the first $n-1$ jobs are on-time.

Lemma 4. If two jobs $2 i$ and $2 i-1$ are both on-time, then there exists $j, 1 \leq j<i$, such that jobs $2 j$ and $2 j-1$ are both late. 
Proof. Suppose by contradiction that there exists a sequence in which for all $j, 0 \leq j<i$, at least one job $2 j$ or $2 j-1$, denoted $a_{[j]}$-job, is on time and that jobs $2 i$ and $2 i-1$ are both on-time.

It is clear that resequencing these on-time jobs in a non-decreasing order of their due dates does not increase the number of late jobs.

In this new sequence, jobs $2 i$ and $2 i-1$ are sequenced last with respect to the considered on-time jobs. The completion time of the last job $(2 i$ or $2 i-1)$ is greater than or equal to the sum of

1. the sum of the minimal processing times of $a_{[j]}$-jobs scheduled before jobs $2 i$ and $2 i-1,0 \leq j<i$, denoted by $A$, and,

2. the processing times of jobs $2 i$ and $2 i-1$, denoted by $B$.

We have

$$
\begin{aligned}
A & =\left\{\begin{array}{l}
a_{1}+ \\
a_{3}+a_{2}+ \\
a_{5}+a_{4}+a_{2}+ \\
\cdots \\
a_{2 i-3}+a_{2 i-4}+\cdots+a_{4}+a_{2}
\end{array}\right. \\
& =a_{1}+a_{3}+\cdots+a_{2 i-3}+a_{2 i-4}+\cdots+(i-2) * a_{2}
\end{aligned}
$$

and

$$
\begin{aligned}
B & =p_{2 i}^{1}+p_{2 i-1}^{1}=p_{2 i}^{2}+p_{2 i}^{2} \\
& =a_{2 i}+a_{2 i-1}+2 * a_{2 i-2}+\cdots+2 * a_{4}+2 * a_{2} .
\end{aligned}
$$

The completion time of the last job is greater than or equal to

$$
\begin{aligned}
A+B & =a_{1}+a_{3}+\cdots+a_{2 i-3}+a_{2 i}+2 a_{2 i-2}+\ldots+i a_{2} \\
& =a_{1}+a_{3}+\cdots+a_{2 i-3}+d_{2 i} \\
& >d_{2 i} .
\end{aligned}
$$

Hence at least one job among jobs $2 i$ and $2 i-1$ is late. This contradicts the considered hypothesis.

Lemma 5. Every schedule admits at most $n$ on-time jobs. 
Proof. Suppose by contradiction that there exists a sequence such that the number of on-time jobs is greater than or equal to $n+1$. Resequencing the on-time jobs in a nondecreasing order of their due dates and sequencing late jobs last does not increase the number of late jobs. Let $\pi$ be such a sequence.

Since we have $n+1$ on-time jobs, then there exist at least an integer $i$ such that jobs $2 i$ and $2 i-1$ are both on-time. Let $i^{*}$ be the smallest $i$ such that $2 i$ and $2 i-1$ are both on-time and $\pi_{i^{*}}$ be the subsequence of on-time jobs among jobs $k, 1 \leq k \leq 2 i^{*}$. We have $\pi=\left(\pi_{i^{*}}, \pi_{R}\right)$. Notice that in subsequence $\pi_{i^{*}}$ there are at most $i^{*}$ jobs, which are all on-time.

Due to Lemma 4, there exists an integer $j, 1 \leq j<i^{*}$, such that neither job $2 j$ nor job $2 j-1$ is on-time. We construct a new subsequence sequence $\pi_{i^{*}}^{\prime}$ in which we exchange one job among $2 i^{*}$ and $2 i^{*}-1$ (which are on-time in $\pi$ ) with job $2 j$ or $2 j-1$ (which are late in $\pi$ ). For example, we exchange jobs $2 i^{*}$ and $2 j-1$. Then, we reorder the jobs of $\pi_{i^{*}}^{\prime}$ in a non decreasing order of their due dates. Due to Lemma 3, all jobs of $\pi_{i^{*}}^{\prime}$ are on-time. Since $\forall s \in S, p_{2 j-1}^{s}<p_{2 i^{*}}^{s}$, then sequence $\left(\pi_{i^{*}}^{\prime}, \pi_{R}\right)$ has at least the same number jobs on-time as $\pi$.

We can reiterate the same reasoning until we get a sequence in which we have at most $n-1$ jobs on-time among jobs $1, \ldots, 2 n-2$. Since we have at least $n+1$ jobs on-time, then jobs $2 n$ and $2 n-1$ are both on-time but this cannot occur due to Lemma 4 .

The following theorem hold.

Theorem 5. Problem MinMax $\left(1|| \sum U_{j}, p_{j}\right)$ is NP-hard even when $|S|=2$.

Proof. We first prove that, if the even-odd partition problem has a solution, then the constructed instance admits a solution with $n$ on-time jobs.

Let $A_{1}$ be a solution of the even-odd partition problem. Consider a sequence $\pi=$ $\left(\pi_{1}, \pi_{2}\right)$ such that

- for all $j \leq 2 n$, if $a_{j} \in A_{1}$ then job $j \in \pi_{1}$;

- the jobs of $\pi_{1}$ are ordered in non-decreasing order of their due dates.

Suppose that $\pi=([1],[2], \ldots,[n],[n+1],[n+2], \ldots[2 n])$ and $\pi_{1}=([1],[2], \ldots,[n])$

Due to Lemma 3, the first $n-1$ jobs in $\pi_{1}$ are on-time. We prove that the last job in $\pi_{1}$ is also on-time.

The completion time of the last job $[n]$ in scenario $s_{1}$ is 


$$
\begin{aligned}
C_{[n]}\left(\pi, s_{1}\right) & =p_{[1]}^{1}+p_{[2]}^{1}+\cdots p_{[n]}^{1} \\
& =\left\{\begin{array}{l}
a_{[1]}+ \\
a_{[2]}+a_{2}+ \\
\cdots \\
a_{[n]}+a_{2 n-2}+\cdots+a_{4}+a_{2}
\end{array}\right. \\
& =a_{[1]}+a_{[2]}+\cdots+a_{[n]}+a_{2 n-2}+\cdots+(n-1) * a_{2} \\
& =B+d_{2 n-2} \\
& =d_{2 n}=d_{2 n-1}=d_{[n]} .
\end{aligned}
$$

The completion time of the last job $[n]$ in scenario $s_{2}$ is

$$
\begin{aligned}
C_{[n]}\left(\pi, s_{2}\right) & =p_{[1]}^{2}+p_{[2]}^{2}+\cdots p_{[n]}^{2} \\
& =\left\{\begin{array}{l}
a_{[n+1]}+ \\
a_{[n+2]}+a_{2}+ \\
\cdots \\
a_{[2 n]}+a_{2 n-2}+\cdots+a_{4}+a_{2}
\end{array}\right. \\
& =a_{[n+1]}+a_{[n+2]}+\cdots+a_{[2 n]}+a_{2 n-2}+\cdots+(n-1) * a_{2} \\
& =B+d_{2 n-2} \\
& =d_{2 n}=d_{2 n-1}=d_{[n]} .
\end{aligned}
$$

Consequently, job $[n]$ is on-time for the two scenarios and the number of on-time jobs in $\pi$ is at least $n$ and cannot be greater than $n$ due to Lemma 5 .

We prove now that if the constructed instance admits a solution with $n$ on-time jobs, then the even-odd partition problem admits a solution.

Let $\pi$ be a sequence in which the number of on-time jobs is equal to $n$. Suppose that in $\pi$, there exist two on-time jobs $2 i$ and $2 i-1$. Then, due to Lemma 4, there exists an index $j, 1 \leq j<i$, such that jobs $2 j$ and $2 j-1$ are both late. Using the same technique as in Lemma 5, we transform sequence $\pi$ in a new sequence in which job $2 j$ or $2 j-1$ is on-time and job $2 i$ or $2 i-1$ is late. We reiterate the same reasoning until we get a sequence $\pi^{\prime}$, in which for all $k, 1 \leq k \leq n$, only one job among jobs $2 k$ and $2 k-1$ is on-time.

Resequencing the on-time jobs in a non-decreasing order of their due dates and sequencing the late jobs last we do not increase the number of late jobs. We have $\pi^{\prime}=([1],[2], \ldots,[n],[n+1],[n+2], \ldots[2 n])$ and jobs $[i], 1 \leq i \leq n$ are on-time. 
The completion time of job $[n]$ in scenario $s_{1}$ is

$$
\begin{aligned}
C_{[n]}\left(\pi^{\prime}, s_{1}\right) & =p_{[1]}^{1}+p_{[2]}^{1}+\cdots p_{[n]}^{1} \\
& =a_{[1]}+a_{[2]}+\cdots+a_{[n]}+a_{2 n-2}+\cdots+(n-1) * a_{2} \\
& =a_{[1]}+a_{[2]}+\cdots+a_{[n]}+d_{2 n-2}
\end{aligned}
$$

The completion time of job $[n]$ in scenario $s_{2}$ is

$$
\begin{aligned}
C_{[n]}\left(\pi^{\prime}, s_{2}\right) & =p_{[1]}^{2}+p_{[2]}^{2}+\cdots p_{[n]}^{2} \\
& =a_{[n+1]}+a_{[n+2]}+\cdots+a_{[2 n]}+a_{2 n-2}+\cdots+(n-1) * a_{2} \\
& =a_{[n+1]}+a_{[n+2]}+\cdots+a_{[2 n]}+d_{2 n-2} .
\end{aligned}
$$

Since job $[n]$ is on-time then

$$
\forall s \in\left\{s_{1}, s_{2}\right\}, C_{[n]}\left(\pi^{\prime}, s\right) \leq d_{[n]}=d_{2 n}=d_{2 n-1}=B+d_{2 n-2} .
$$

Consequently, we have

$$
\left\{\begin{array}{l}
a_{[1]}+a_{[2]}+\cdots+a_{[n]} \leq B \\
a_{[n+1]}+a_{[n+2]}+\cdots+a_{[2 n]} \leq B, \text { and } \\
a_{[1]}+a_{[2]}+\cdots+a_{[2 n]}=B
\end{array}\right.
$$

This proves that

$$
a_{[1]}+a_{[2]}+\cdots+a_{[n]}=a_{[n+1]}+a_{[n+2]}+\cdots+a_{[2 n]}=B,
$$

which means that the even-odd partition problem has a solution.

Correspondingly. the following corollary also holds.

Corollary 3 Problem MinMax $\left(1|| \sum U_{j}, p_{j}, d_{j}\right)$ is NP-hard even when $|S|=2$.

\section{References}

[1] I. Averbakh, Minmax regret solutions for minimax optimization problems with uncertainty, Operations Research Letters, 27, 57-65, 2000. 
[2] K.R. Baker, Introduction to Sequencing and Scheduling, John Wiley and Sons, New York, NY, 1974.

[3] R.L. Daniels, P. Kouvelis, Robust scheduling to hedge against processing time uncertainty in single stage production, Management Science 41: 363-376, 1995.

[4] M.R. Garey and D.S. Johnson, Computers and Intractability: A Guide to the Theory of NP-Completeness, W. H. Freeman, 1979.

[5] R.L. Graham, E.L. Lawler, J.K. Lenstra and A.H.G. Rinnooy Kan, Optimization and approximation in deterministic machine scheduling: a survey, Annals of Discrete Mathematics 5: 287-326, 1979.

[6] A. Kasperski, Minimizing maximal regret in the single machine sequencing problem with maximum lateness criterion, Operations Research Letters, 33, 431-436, 2005.

[7] P. Kouvelis and R.L. Daniels and G. Vairaktarakis, Robust scheduling of a twomachine flow shop with uncertain processing times, IIE Transactions, 32: 421-432, 2000 .

[8] P. Kouvelis and G. Yu, Robust Discrete Optimisation and its Applications, Kluwer Academic Publisher, 1997.

[9] E.L. Lawler, Optimal sequencing of a single machine subject to precedence constraints, Management Science 19: 544-546, 1973

[10] J.M. Moore, A $n$ job, one machine sequencing algorithm for minimizing the number of late jobs, Management Science 15: 102-109, 1968.

[11] W.E. Smith, Various optimizers for single-stage production, Naval Research Logistics Quarterly 3 (1956) 59-66.

[12] J. Yang and G. Yu, On the Robust Single Machine Scheduling Problem, Journal of Combinatorial Optimization, 6, 17-33, 2002. 